\title{
Proposta de um CubeSat universitário para desenvolvimento de tecnologias espaciais nacionais
}

\author{
Eduardo Henrique da Silva ${ }^{1}$, João Luiz Dallamuta Lopes ${ }^{2}$ \\ ${ }^{1}$ Universidade Tecnológica Federal do Paraná, Cornélio Procópio, PR, Brasil \\ Aluno de Graduação do curso de Engenharia Eletrônica. \\ ${ }^{2}$ Instituto Nacional de Pesquisas Espaciais, São José dos Campos, SP, Brasil \\ Aluno de Doutorado do curso de Engenharia e Tecnologias Espaciais - ETE. \\ ehenriquesilva05@gmail.com
}

\begin{abstract}
Resumo. O crescimento expressivo de pequenos satélites na última década gerou oportunidades para novos fornecedores de tecnologias espaciais e modelos de negócios envolvendo essa classe de satélites. No Brasil esta tendência não passou despercebida, com o lançamento de algumas missões destinadas a validação de tecnologias desenvolvidas localmente ou ao desenvolvimento de aprendizado sobre integração e operação de pequenos satélites. O lançamento de CubeSats deve seguir em alta na próxima década e isto é uma oportunidade para desenvolvimento de tecnologias nacionais para esta classe de missão. Este artigo propõe um CubeSat de 2 U cuja carga útil são slots para a validação em órbita de subsistemas projetados por universidades e empresas brasileiras que tenham por objetivo competir como fornecedores de tecnologia para este tipo de satélite. Uma arquitetura preliminar de missão é proposta bem como suas expectativas de custos.
\end{abstract}

Palavras-chave: CubeSat; Universidades; Desenvolvimento de Tecnologia.

\section{Introdução}

Um CubeSat é um cubo de $10 \mathrm{~cm}$ com massa de até $1,33 \mathrm{~kg}$. Iniciado em 1999, o Projeto CubeSat começou como um esforço colaborativo entre o Prof. Jordi PuigSuari na Universidade Politécnica da Califórnia (Cal Poly), San Luis Obispo e o Prof. Bob Twiggs no Laboratório de Desenvolvimento de Sistemas Espaciais da Universidade de Stanford (SSDL). O propósito do projeto é fornecer um padrão para o design de picosatélites para reduzir custos 
e tempo de desenvolvimento, aumentar a acessibilidade ao espaço e sustentar lançamentos frequentes. [MEHRPARVAR, 2014].

Entre 1998 e 2019 foram lançadas mais de 1300 missões com massa inferior a 10kg, em sua expressiva maioria missões baseadas no padrão CubeSat. Entre 2020 e 2023 cerca de 1990 missões do mesmo tipo estão planejadas. [KULI, 2020]. A figura 1 ilustra a evolução dos lançamentos de nanosatélites desde 1998. Os dados de 2020 até 2023 são estimativas baseadas em missões em desenvolvimento. Esta estimativa não considera os impactos que a pandemia de COVID-19 pode resultar em calendários de missões.

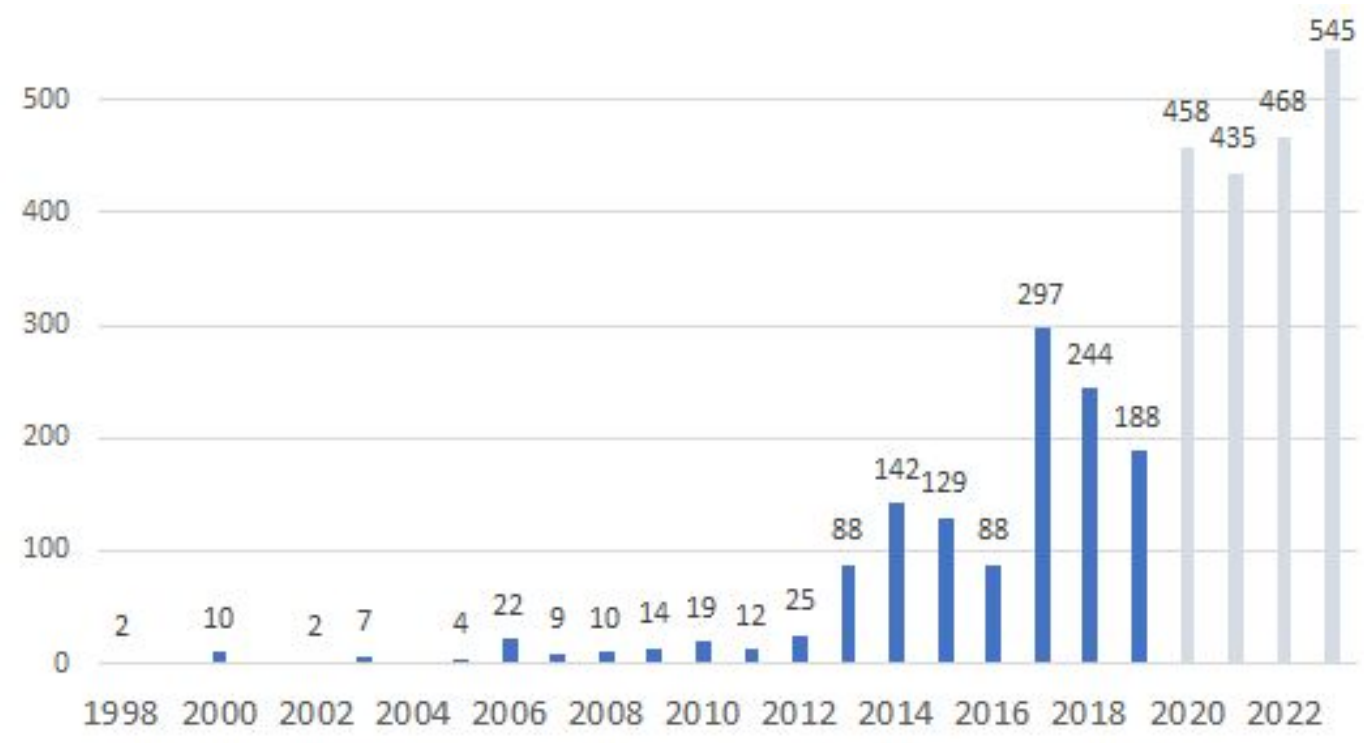

Figura 1. Missões de nanosatélites 1998 - 2023. [Fonte: KULI, 2020]

Os Estados Unidos são indiscutivelmente os precursores da utilização de nanosatélites em missões espaciais, tendo colocado em órbita um total de 473 até o mês de abril de 2017, possuindo um percentual de $76 \%$ de todos os CubeSats já lançados. Além disso, são líderes nas contribuições técnico-científicas publicadas em anais de congressos e periódicos. [KREBS, 2017]. Esses números refletem diretamente o nível de investimento do país no setor, fomentando a pesquisa e desenvolvimento $(\mathrm{P} \& \mathrm{D})$ e gerando benefícios à economia nacional.

O início da inclusão dos CubeSats na frota brasileira se deu em meados de 2014, com o NanoSatC-Br1 sendo o primeiro CubeSat brasileiro a ser enviado ao espaço. Foi desenvolvido pelo Instituto Nacional de Pesquisas Espaciais (INPE) em parceria com a Universidade Federal de Santa Maria (UFSM), e sua missão era a validação de tecnologias de circuitos integrados projetados no país, o estudo da Anomalia Magnética do Atlântico Sul e a capacitação e treinamento de estudantes. [VILLELA, 2016]. A tabela 1 mostra todas as missões brasileiras abaixo de $13 \mathrm{~kg}$ já lançadas. 
Tabela 1. Satélites brasileiros enviados ao espaço. [Fonte: Space Launch Report, www.spacelaunchreport.com; Websites dos projetos]

\begin{tabular}{|c|c|c|c|c|c|}
\hline Satélite & Integrator & Massa & Lançamento & Carga útil & $\begin{array}{l}\text { Final da } \\
\text { Operação }\end{array}$ \\
\hline $\begin{array}{l}\text { Dove-OSCA } \\
\text { R } 17\end{array}$ & AMSAT-BR & $12,92 \mathrm{~kg}$ & 1990 & $\begin{array}{l}\text { Sintetizador de } \\
\text { voz }\end{array}$ & 1998 \\
\hline $\begin{array}{l}\text { NanoSatC-Br } \\
1\end{array}$ & INPE-UFSM & $1 \mathrm{~kg}$ & 2014 & $\begin{array}{l}\text { Circuitos } \\
\text { integrados } \\
\text { endurecidos } \\
\text { contra radiação }\end{array}$ & Ativo \\
\hline AESP-14 & ITA & $1 \mathrm{~kg}$ & 2015 & $\begin{array}{l}\text { Sonda de } \\
\text { Langmuir }\end{array}$ & $\begin{array}{c}\text { Falha em } \\
\text { órbita }\end{array}$ \\
\hline SERPENS & INPE & $3 \mathrm{~kg}$ & 2015 & $\begin{array}{l}\text { Transponder e } \\
\text { um Impulsor de } \\
\text { Plasma }\end{array}$ & 2016 \\
\hline Tancredo 1 & $\begin{array}{l}\text { Escola } \\
\text { Tancredo } \\
\text { Neves }\end{array}$ & $0,67 \mathrm{~kg}$ & 2017 & $\begin{array}{l}\text { Gravador de } \\
\text { Voz/ Sonda de } \\
\text { Langmuir }\end{array}$ & 2018 \\
\hline ITASAT-1 & ITA & $8 \mathrm{~kg}$ & 2018 & $\begin{array}{l}\text { Experimental } \\
\text { Transponder/ } \\
\text { 80m camera/ } \\
\text { GPS Receiver }\end{array}$ & Ativo \\
\hline FloripaSat-1 & UFSC & $1 \mathrm{~kg}$ & 2019 & $\begin{array}{l}\text { Rádio Amador } \\
\text { Transponder/ } \\
\text { Experimentos } \\
\text { FPGA }\end{array}$ & Ativo \\
\hline
\end{tabular}

O histórico brasileiro nesta classe de missão demonstra a capacidade e interesse no desenvolvimento de tecnologias nacionais embarcadas na forma de subsistemas e cargas úteis. Este desenvolvimento está concentrado nas universidades e institutos de pesquisas. Porém a capacidade nacional de geração de spin-offs, empresas privadas derivadas do desenvolvimento tecnológico, ainda não ocorreu. Este artigo tem como objetivo propor em um nível preliminar uma missão espacial na faixa de $2 \mathrm{~kg}$ destinada a demonstração em órbita de subsistemas desenvolvidos por instituições brasileiras governamentais ou privadas, com potencial de geração de produtos comerciais. 


\section{Metodologia}

A metodologia empregada para este trabalho se inicia com uma consulta às referências bibliográficas do tema, quanto a CubeSat, seus ecossistemas industriais, bem como as principais bases de dados sobre esta classe de missão. Situada a importância da pesquisa, um modelo de missão em fase inicial de análise técnica e financeira é proposto, considerando valores de referência encontrados nos trabalhos científicos pesquisados.

\section{Resultados e Discussão}

O CubeSat proposto neste trabalho contará com uma estrutura $2 \mathrm{U}(10 \times 10 \times 20 \mathrm{~cm})$ e será composto por um módulo de serviço, composto pelos subsistemas responsáveis pela operação do satélite, e por um módulo de carga útil, que conterá slots para experimentos de validação de tecnologias nacionais. A figura 2 ilustra, por meio de um diagrama de blocos, todos os subsistemas existentes no módulo de serviço do CubeSat sugerido, assim como o módulo de carga útil.

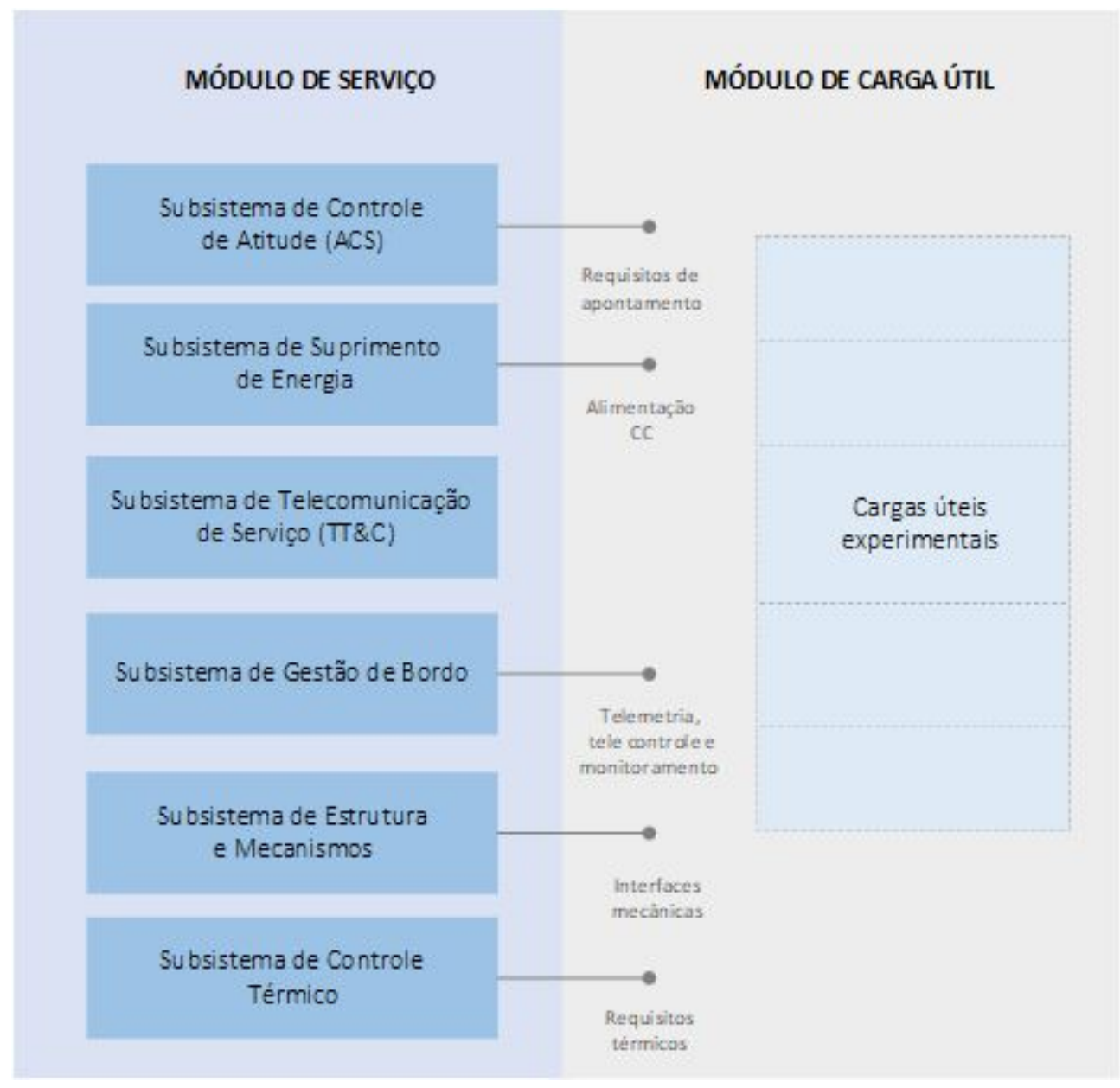

Figura 2. Diagrama de blocos do CubeSat proposto. 
Nos tópicos a seguir, cada subsistema e suas respectivas funções são explicados brevemente:

- Subsistema de Gestão de Bordo: tem como principal função o gerenciamento e controle das funções do nanosatélite, realizando o processamento e armazenamento dos dados originários dos demais subsistemas. [ALVES, 2019].

- Subsistema de Telecomunicações de Serviço (TT\&C): tem a função de comunicar a estação terrestre com o CubeSat, transmitindo e recebendo, respectivamente, pacotes de telemetria e de telecomando. [HEUNIS, 2014].

- Subsistema de Suprimento de Energia: é responsável por todo o fornecimento, armazenamento e distribuição de energia entre todos os subsistemas. É de importância vital para o nanosatélite pois realiza também a geração de energia, que ocorre geralmente a partir de painéis fotovoltaicos. [PAULA, 2019].

- Subsistema de Controle Térmico: é o subsistema responsável pelo controle e gerenciamento da temperatura de todos os componentes que compõem o CubeSat. [GILMORE, 1994].

- Subsistema de Estrutura e Mecanismos: comporta todos os subsistemas e garante que nenhuma parte do nanosatélite sofra danos que possam resultar no fracasso da missão, assim como protege os componentes eletrônicos contra a radiação ionizante existente em ambiente espacial. [ADDAIM, 2010].

- Subsistema de Controle de Atitude: é fundamental para o alinhamento correto de painéis solares e antenas presentes no nanosatélite, além de ser responsável pelo apontamento de câmeras direcionadas à Terra, quando presentes no CubeSat. [JULIO FILHO, 2019].

- Carga útil: este módulo será responsável pela validação de tecnologias espaciais nacionais, que serão inseridas em slots do CubeSat para a execução da missão espacial proposta.

O CubeSat proposto terá 1U (seção superior) para o módulo de serviço, onde estarão todos os subsistemas citados anteriormente, e 1U (seção inferior) para a carga útil, composta por tecnologias nacionais a serem validadas em órbita. A figura 3 ilustra o modelo do CubeSat $2 \mathrm{U}$ sugerido, desenvolvido na versão de estudante do AutoCAD, software utilizado para a criação de projetos de engenharia. 


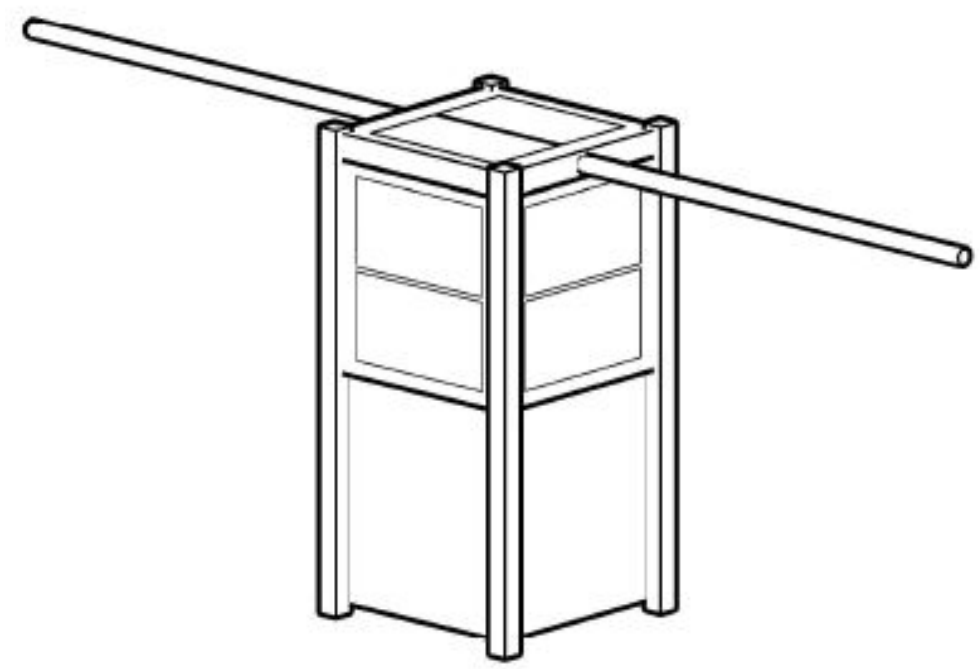

Figura 3. Modelo sugerido do CubeSat 2U.

A seleção das cargas úteis para a missão obedece às limitações de três variáveis de projeto que possam ser providas pelo módulo de serviço, sendo elas: Potência elétrica requerida, necessidade de transferência de dados e interfaces mecânicas.

\section{Aspectos de potência elétrica disponível para a carga útil}

Os consumos estimados por carga do módulo de serviço é descrito na tabela 2, são considerados valores de referência propostos por [CLARK e LOGAN, 2011] considerando um transceptor de $\mathrm{VHF} / \mathrm{UHF}$.

Tabela 2. Cargas elétricas do módulo de serviço.

\begin{tabular}{lccc}
\hline \multicolumn{1}{c}{ Carga elétrica } & $\begin{array}{c}\text { Potência } \\
(\mathbf{W})\end{array}$ & $\begin{array}{c}\text { Ciclo } \\
\text { por órbita(\%) }\end{array}$ & $\begin{array}{c}\text { Potência média por } \\
\text { órbita }(\mathbf{W})\end{array}$ \\
\hline $\mathrm{TX}$ & 1,9 & $10 \%$ & 0,19 \\
$\mathrm{RX}$ & 0,15 & $100 \%$ & 0,15 \\
Magnetorquers & 0,1 & $2 \%$ & 0,002 \\
Sensores & 0,001 & $100 \%$ & 0,001 \\
Computador de bordo & 0,25 & $100 \%$ & 0,25 \\
Aquecedor de bateria & 0,2 & $20 \%$ & 0,04 \\
Sistema de potência & 0,005 & $100 \%$ & 0,005 \\
\multicolumn{2}{c}{ Potência média por órbita com margem de $\mathbf{1 5 \%}$} & $\mathbf{0 , 7 3 3 7} \mathbf{~ W}$ \\
\hline
\end{tabular}




\section{Workshop em Engenharia e Tecnologia Espaciais \\ 18, 19 e 20 de agosto de 2020}

Considerando células com $27 \%$ de eficiência ao final da vida orbital, com os painéis solares cobrindo $1 \mathrm{U}$ do satélite destinado ao módulo de serviço, conforme ilustrado na figura 3, a capacidade de geração média é estimada em $2 \mathrm{~W}$ por órbita. Considerando $15 \%$ de perdas de conversão e armazenagem, a potência média por órbita ou Orbit Average Power (OAP) é de $1,7 \mathrm{~W}$, considerando o estimado de carga para o módulo de serviço, a capacidade disponível para as cargas úteis é de $0,97 \mathrm{~W}$ médios por órbita.

\section{Aspectos de capacidade de transmissão e recepção disponíveis para a carga útil}

O módulo transceptor proposto para o módulo de serviço utiliza da faixa VHF/UHF para uplink e downlink respectivamente, em protocolo X25. Esta configuração usual em projetos de CubeSat utiliza-se de frequências de radioamadores e visa baratear o segmento solo do projeto bem como aumentar o número de estações que podem rastrear o satélite. $\mathrm{O}$ desempenho padrão deste tipo de transceptor é de 9600 bps para transmissão e recepção em regime full duplex. Para um período de 9 minutos por órbita de comunicação, o total transferido e recebido é de $5,18 \mathrm{Mb}$. Considerando uma divisão igualitária de dados entre o módulo de serviço e a carga útil, podem ser transmitidos até $2,59 \mathrm{Mb}$ de dados por órbita pela carga útil.

Experimentos de cargas úteis que requeiram maior capacidade de transmissão, como imageadores, precisam de enlaces com maiores taxas de transferência, normalmente em banda $\mathrm{S}$, sendo o consumo e espaço físico deste transceptor alocados ao módulo de carga útil.

\section{Aspectos físicos de carga útil (massa, estruturas e mecanismos)}

A carga útil será alocada no espaço de $1 \mathrm{U}$, conforme as descrições das figuras 2 e 3 . As limitações globais de carga útil são portanto $1000 \mathrm{~cm}^{3}$ de volume e $1 \mathrm{~kg}$ de massa. Aspectos ligados a políticas de lançamento para esta classe de espaçonaves também fazem parte das limitações da carga útil, como ausência de materiais inflamáveis e de vasos pressurizados. Experimentos que requerem interfaces externas, como antenas, sensores, painéis solares possuem as áreas do módulo $1 \mathrm{U}$ para fixação.

\section{Custos do satélite e lançamento}

Os custos envolvidos no desenvolvimento e lançamento de um CubeSat são significativamente menores do que os investidos em satélites de médio e grande porte. Isso ocorre devido às suas dimensões reduzidas, além do fato de serem utilizados para um único propósito. [WOELLERT, 2010].

A estimativa de gastos para a construção do nanosatélite foi realizada a partir da cotação dos componentes necessários para cada subsistema, embasando-se no website CubeSat Shop e no modelo de custos AMES para Micro/Nanosatélites. [PAINE, 2015]. Na tabela 3 abaixo, os custos relativos à cada subsistema são apresentados em euros $(€)$, seguindo a cotação do dia 01 de julho de 2020 obtida no website UOL Economia, e sua respectiva conversão em reais $(\mathrm{R} \$)$. 
Tabela 3. Cotação dos subsistemas. [Fonte: CubeSatShop, www.cubesatshop.com; UOL Economia, www.economia.uol.com.br]

\begin{tabular}{lcc}
\hline \multicolumn{1}{c}{ Subsistema } & Custo $(\boldsymbol{€})$ & Custo $(\mathbf{R} \mathbf{\$})$ \\
\hline Gestão de Bordo & $12.600,00$ & $75.348,00$ \\
Telecomunicações de Serviço & $13.750,00$ & $82.225,00$ \\
Suprimento de Energia & $11.850,00$ & $70.863,00$ \\
Controle Térmico & $4.550,00$ & $27.209,00$ \\
Estrutura e Mecanismos & $3.150,00$ & $18.837,00$ \\
Controle de Atitude & $37.350,00$ & $223.353,00$ \\
Total & $\mathbf{8 3 . 2 5 0 , 0 0}$ & $\mathbf{5 0 0 . 8 3 5 , 0 0}$ \\
\hline
\end{tabular}

Com relação ao lançamento, existem instituições privadas que oferecem esse tipo de serviço para satélites miniaturizados, como a Rocket Labs, empresa norte-americana especializada no projeto e construção de foguetes. $\mathrm{O}$ valor médio cobrado para colocar um CubeSat em órbita atualmente gira em torno de US $\$ 100$ mil para cada $1 \mathrm{U}$, portanto, o custo total de lançamento do nanosatélite proposto neste trabalho é de aproximadamente US\$200 mil.

\section{Conclusão}

Este artigo propôs em um nível preliminar um CubeSat de $2 \mathrm{U}$ destinado a validação em órbita de subsistemas projetados por universidades e empresas brasileiras que tenham por objetivo a oferta comercial desta tecnologia no mercado global de pequenas missões espaciais.

Aspectos técnicos iniciais da missão foram delineados bem como um orçamento de custo para a montagem do satélite e seu lançamento.

Em uma fase posterior, uma análise da missão em ambiente computacional e o detalhamento dos requisitos da espaçonave e das cargas úteis candidatas serão emitidos, bem como o refinamento de orçamento e cronograma da missão.

\section{Referências}

Addaim, A.; Kherras, A.; Zantou, E. B. (2010) "Design of Low-cost Telecommunications CubeSat-class Spacecraft" In: Aerospace Technologies Advancements, Edited by Thawar T. Arif, IntechOpen, DOI: 10.5772/6925. Disponível em: $<$ https://www.intechopen.com/books/aerospace-technologies-advancements/design-of-low-co st-telecommunications-cubesat-class-spacecraft> 


\section{Workshop em Engenharia e Tecnologia Espaciais \\ 18,19 e 20 de agosto de 2020}

Alves, A. C. R.; Dias, S. M.; Queiroz, K. I. P. M.; de Carvalho, M. J. M.; Duarte, J. M. L. (2019) "CONASAT-0: Visão Geral do Nanossatélite Desenvolvido." In: II Congresso Aeroespacial Brasileiro, 2019, São José dos Campos. ANAIS - CAB 2019.

Clark, C. and Logan, R. (2011) "Power Budgets for Mission Success." CalPoly.

Franke, L. L. C.; Farias, T. T.; Balestrin, M. R.; Silveira, I. C.; Jaenisch. G.P.; Piaz, M. A. D.; Durão, O. S. C.; Schuch, N. J. (2014) "Simulação Térmica do primeiro Cubesat brasileiro: NANOSATC-BR1." In: Congresso Nacional de Estudantes de Engenharia Mecânica, Rio de Janeiro. ANAIS - CREEM 2014.

Gilmore, D. G. (1994) "Satellite Thermal Control Handbook". 2a ed. Aerospace Corporation, Califórnia, EUA.

Heunis, A. E. (2014) "Design and implementation of generic flight software for a cubesat" Dissertação de mestrado, Department Electrical and Electronic Engineering, Stellenbosch University, África do Sul.

Julio Filho, A. C.; Tikami A.; Paula, E. S. F.; Murcia, J.; Fernandes, G. F.; Camargo, L. P.; Santos, C. A. M. B.; Santos, W. A.; Naccarato, K. (2019) "Desenvolvimento de um CubeSat para detecção de descargas atmosféricas: Projeto RaioSat" In: II Congresso Aeroespacial Brasileiro, São José dos Campos. ANAIS - CAB 2019.

Krebs, G. (2017) "Spacecraft by country." In: Gunter Space Page Disponível em: $<$ http://space.skyrocket.de/directories/sat_c.htm>

Kuli, E. Nanosats Database (2020). Disponível em: <https://www.nanosats.eu>. Acesso em: 31 de maio de 2020.

Mehrparvar, A. (2014) "CubeSat Design Specification" The CubeSat Program, CalPoly SLO.

Paine, T.; Saing, M. S. C.; Hamilton, B. A. (2015) “Ames Micro/NanosatellitES Cost Model”. In: 2015 NASA Cost Symposium.

Paula, E. S. F.; Magalhães, R. O. (2019) "Projeto Preliminar de Painel Solar e Dimensionamento de Bateria de um CubeSat 3U para Detecção de Raios”. In: X Workshop em Engenharia e Tecnologias Espaciais, São José dos Campos. ANAIS - WETE 2019.

Villela, T.; Brandão, A. M.; Leonardi, R. (2016). "Cubesats e oportunidades para o setor espacial brasileiro". In: Parcerias Estratégicas, Brasília-DF, v. 21, n. 42, p. 91-114. jan-jun 2016

Woellert, K.; Ehrenfreund, P.; Ricco A. J.; Hertzfeld H. (2010) "Cubesats: Cost-effective science and technology platforms for emerging and developing nations". In: Advances in Space Research. 